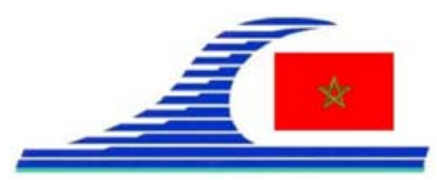

Conférence Méditerranéenne Côtière et Maritime EDITION 2, TANGER, MAROC (2011)

Coastal and Maritime Mediterranean Conference

Disponible en ligne - http://www.paralia.fr - Available online

\title{
Caractérisations géotechnique et mécanique des sédiments marins de dragage du port de Concarneau (France)
}

\section{Lionel MANCIOPPI ${ }^{1}$, Daniel LEVACHER ${ }^{2}$, Mickaël BERTRAND ${ }^{1,2}$, Philippe DHERVILLY ${ }^{1}$, Chengcheng XU ${ }^{1,2}$}

1. SEDIGATE S.A.S., 17 rue Claude Bloch, BP 25 102, 14000 Caen Cedex 05, France. l.mancioppi@sedigate.com ; contact@sedigate.com

2 Université de Caen, Laboratoire de Morphodynamique Continentale et Côtière, M2C UMR 6143, 24 rue des Tilleuls, 14000 Caen, France.

daniel.levacher@unicaen.fr

\section{Résumé :}

En France, chaque année les opérations de dragage dans les ports, les voies navigables et les plans d'eau génèrent un volume estimé à 55 millions de $\mathrm{m}^{3}$. La réglementation française implique une gestion à terre à partir de niveaux N1 et N2 déterminés par le groupe GEODE faisant seuils de référence dans l'arrêté du 06/08/2006 du Code de l'Environnement. Les caractérisations géotechnique et mécanique présentées font partie de travaux qui s'inscrivent dans une gestion à terre de sédiments issus du dragage du port de Concarneau, situé dans le département du Finistère (29) en France. Cette étude est une réponse partielle à un appel d'offre des marchés publics français proposant une valorisation des sédiments en remplissage de zone de stockage, à l'aide de géotextiles et de géomembranes. Tout d'abord, l'étude concerne la détermination des paramètres de base géotechniques des sédiments bruts, paramètres constituant la fiche d'identité des sédiments à valoriser. L'analyse de ces propriétés géotechniques permet d'établir un protocole de solidification/stabilisation adapté à base de liants pouzzolaniques ou hydrauliques. Ainsi diverses formulations pour des mélanges sédiments-liants-additifs sont proposées. Des essais en compression simple sur éprouvettes cylindriques viennent valider les formulations mises en œuvre pour répondre aux exigences du Cahiers des Clauses Techniques Particulières (CCTP) dans le cadre d'une gestion à terre des sédiments du dragage du port de Concarneau (29). Les résultats sont analysés et discutés.

\section{Mots-clés :}

Sédiments portuaires - Gestion à terre - Propriétés physico-chimiques - Liants pouzzolaniques - Ciment - Résistance mécanique - Stabilisation/solidification Valorisation

\section{Introduction}

Les sédiments issus du dragage du port de Concarneau proviennent d'un bassin portuaire dénommé bassin de Moros. Les volumes dragués sont estimés à $8000 \mathrm{~m}^{3}$. Ces 
La connaissance de la Mer :

un vecteur du développement durable en Méditerranée

sédiments sont considérés comme étant non immergeables compte tenu de leur pollution en teneur en métaux traces et composés organiques. Le CCTP de l'appel d'offre du port de Concarneau préconise une valorisation de ces sédiments par remplissage d'une zone de stockage à l'aide de géomenbrane et de géotextile. Le CCTP recommande l'utilisation de liant hydraulique dosé à un pourcentage de $20 \%$ (masse de sédiment humide) pour $1 \mathrm{~m}^{3}$ de sédiments dragués avec une siccité de $50 \%$. Pour des impératifs économiques, l'étude de valorisation menée sur ces sédiments propose l'utilisation de liants pouzzolaniques à un dosage bien en deçà des $20 \%$ pour des résistances mécaniques équivalentes.

\section{Matériels et méthodes}

Les essais et mesures effectués dans le cadre de ces travaux l'ont été en respectant les normes françaises en vigueur.

\subsection{Identification des sédiments}

La connaissance des propriétés physico-chimiques de base a pour objectif non seulement l'identification des sédiments mais elle permet d'optimiser les formulations élaborées à base de liants pouzzolaniques ou ajout de ciment, en tenant compte des paramètres géotechniques. La quantité de matière organique $M O$ et la proportion d'argiles peuvent être néfastes au procédé de stabilisation/solidification. Une évaluation de ces paramètres d'identification est donc nécessaire. Elle se fait à partir d'essais géotechniques qui concernent la détermination de la teneur en eau initiale moyenne $W_{i}$, les limites de liquidité $W_{L}$, de plasticité $W_{P}$, l'indice de plasticité $I_{P}$, la nature et distribution granulaire par tamisage sur colonnes pour la partie grossière et granulométrie laser pour la partie fine, la teneur en matière organique $M O$ par la méthode de la perte au feu et la teneur en métaux lourds.

\subsection{Formulations et essai mécanique de validation}

La stabilisation/solidification des sédiments est étudiée à partir de mélanges sédimentliant-additif. Les teneurs en liant et additifs sont exprimées en pour-cent de sédiment humide. Un ajout de liant pouzzolanique a été proposé à faible dosage en remplacement de l'addition de ciment. De nombreuses études relatives à l'utilisation de ces liants pouzzolaniques en vue d'une valorisation de sédiments marins en technique routière ont déjà abouti à de bonnes performances en laboratoire et sur site (BEHMANESH, 2008, SILITONGA et al., 2010, MIRAOUI, 2010, OBANA et al., sous presse). Des traitements à base de ciment ont aussi fait l'objet d'études montrant qu'un fort dosage donne des résistances mécaniques élevées (BOUTOUIL, 1998) mais que du point de vue économique, un taux de 7\% de ciment constitue une limite recommandée (COLIN, 2003). Le liant pouzzolanique considéré est une cendre volante silico-alumineuse 
stabilisant les sables et les sédiments de manière durable. L'additif utilisé est un hydroxyde de calcium, la chaux. Ce produit est utilisé pour réduire la matière organique $M O$ et la teneur en eau. Ainsi différentes formulations de mélange ont été suggérées, testées et comparées à la formulation de base, constituée de $20 \%$ de ciment. Ces mélanges ont donné lieu à la confection d'éprouvettes cylindriques, de $34 \mathrm{~mm}$ de diamètre et d'un élancement de 2. La teneur en eau des sédiments incorporés dans les mélanges était de 100\%. Conformément aux durées conventionnelles de conservation de 7, 14 et 28 jours, des essais de résistance en compression simple ont été réalisés pour l'ensemble des mélanges étudiés, tableau 1. La résistance mécanique en compression $R c$ est l’indicateur retenu pour évaluer et juger de la pertinence des formulations proposées.

Tableau 1. Formulations étudiées pour les sédiments bruts de Concarneau.

\begin{tabular}{llll}
\hline Références & Liant pouzzolanique (\%) & Liant hydraulique (\%) & Additif (\%) \\
\hline Formulation 1 & 10 & 0 & 4 \\
Formulation 2 & 8 & 2 & 4 \\
Formulation 3 & 7 & 3 & 4 \\
Formulation de base & 0 & 20 & 0 \\
\hline
\end{tabular}

Dosages exprimés en pour-cent de masse de sédiment humide.

\section{Résultats et analyses}

\subsection{Caractérisation géotechnique}

Les résultats présentés dans le tableau 2 montrent des teneurs anormalement élevées : une teneur en eau moyenne de $261 \%$ (liée au moyen de prélèvement) et une forte teneur en matière organique de $15 \%$. Ces teneurs sont nuisibles à la stabilisation de ces sédiments par liants hydrauliques, sans traitement préalable. Il faut soit abaisser la teneur en eau soit diminuer la teneur en $M O$. Rappelons que la présence de métaux lourds pose aussi problème, les teneurs dépassant le seuil N1 concernent l'arsenic, le nickel et le plomb. Les teneurs dépassant N2 intéressent le cuivre et le zinc.

Tableau 2. Données géotechniques de base du sédiment brut.

\begin{tabular}{ll}
\hline Paramètres et unités & Valeurs moyennes \\
\hline Teneur en eau initiale (\%) & $W_{i}=261$ \\
\hline Matière organique (\%) & $M O=15$ \\
\hline Limites d'Atterberg (\%) & Limite de liquidité $W_{L}=118$ \\
& Indice de plasticité $\mathrm{Ip}=59$ \\
\hline & Argiles $0<d<2 \mu \mathrm{m}=3$ \\
& Limons $2<d<63 \mu \mathrm{m}=65$ \\
& Sable $63 \mu \mathrm{m}<\mathrm{d}<2 \mathrm{~mm}=32$ \\
\hline
\end{tabular}


La connaissance de la Mer :

un vecteur du développement durable en Méditerranée

L’indice de plasticité $I_{p}$ atteint 59\%, cette valeur permet de classifier les sédiments du port de Concarneau comme un matériau très plastique.

L'analyse granulométrique montre un sédiment majoritairement constitué d'une fraction granulométrique inférieure à $63 \mu \mathrm{m}(68 \%)$. Les proportions de sable $(63 \mu \mathrm{m}<d<2 \mathrm{~mm})$ atteignent 32\%, voir figure 1.

\subsection{Approche d'une formulation}

Tout d'abord des mélanges ciment-sédiment ont été réalisés à teneur initiale, soit 261\% avec les dosages du tableau 1 , qui du point de vue économique évitait toute déshydratation. Le comportement observé après traitement fait apparaître un taux de fissuration important résultant du séchage du mélange comme sur la figure 2 , et de déformation. Une même série de mélanges a donc été proposée après réduction de la teneur en eau à $100 \%$. Une période de maturation sédiment-chaux a été considérée entre 0 et $48 \mathrm{~h}$ pour étudier le rôle de la chaux sur la teneur en eau et sur les matières organiques. Pour une teneur en eau de $80 \%$ et un dosage en chaux de $4 \%$, entre 0 h et $48 \mathrm{~h}$, la réduction est de $5 \%$, cet abaissement reste faible pour une durée d'attente de deux jours qui du point de vue pratique n’est pas négligeable. Les mélanges ont donc été réalisés sans période de maturation. La formulation de base avec $20 \%$ de ciment n’est pas économique, un taux de 7 à 8\% constitue une limite (FOUCHER, 2003).
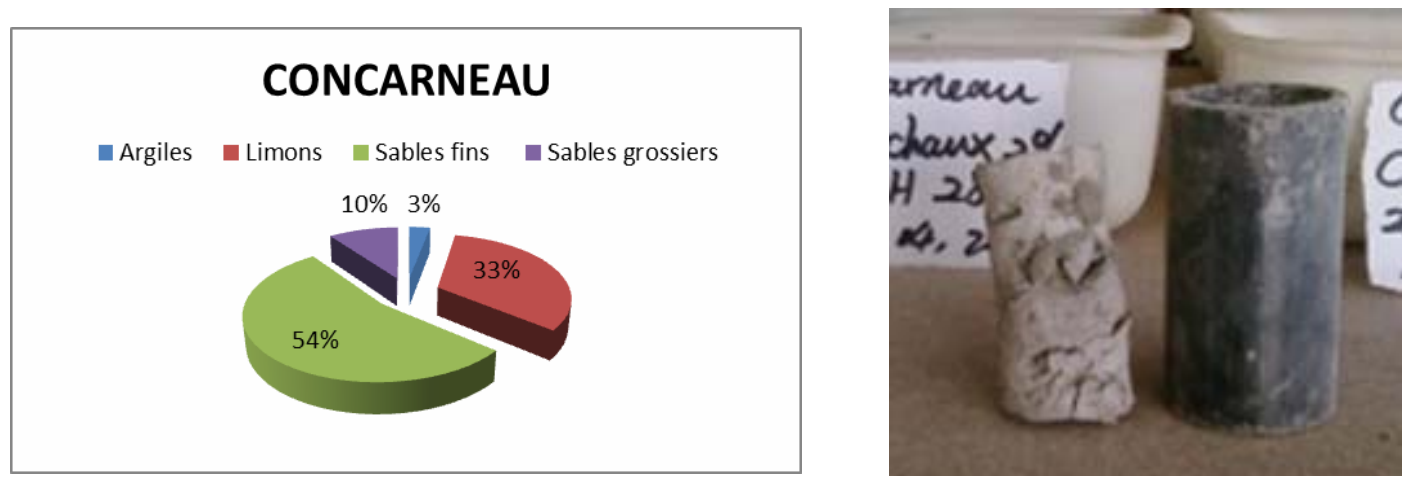

Figure 1. Nature granulométrique des sédiments. Figure 2. Eprouvette endommagée.

\subsection{Choix et validation d'une formulation}

Dans les propositions de formulations, il a été retenu la chaux comme pouvant agir sur la matière organique (REY, 1999) et la substitution du ciment par un liant pouzzolanique (SILITONGA et al., 2008, 2009, 2010). Ce liant pouzzolanique est un mélange de cendres volantes, disponible au niveau industriel, référencé sous le nom d'ACTi100. Le principe a été de diminuer le dosage initial de 10\% en cendres volantes tout en augmentant le pourcentage de ciment et ce, à dosage constant pour la chaux. Les formulations retenues sont données dans le tableau 1. La figure 3 en illustre les performances mécaniques. On observe que l'évolution des résistances à la compression 
suit une tendance classique présentant un seuil ou une tendance asymptotique entre 14 et 28 jours pour les formulations. La combinaison d'un liant pouzzolanique et de ciment avec un taux de chaux constant (4\%) permet d'atteindre une résistance $R c_{28}$ équivalente à la formulation de base, voire plus.

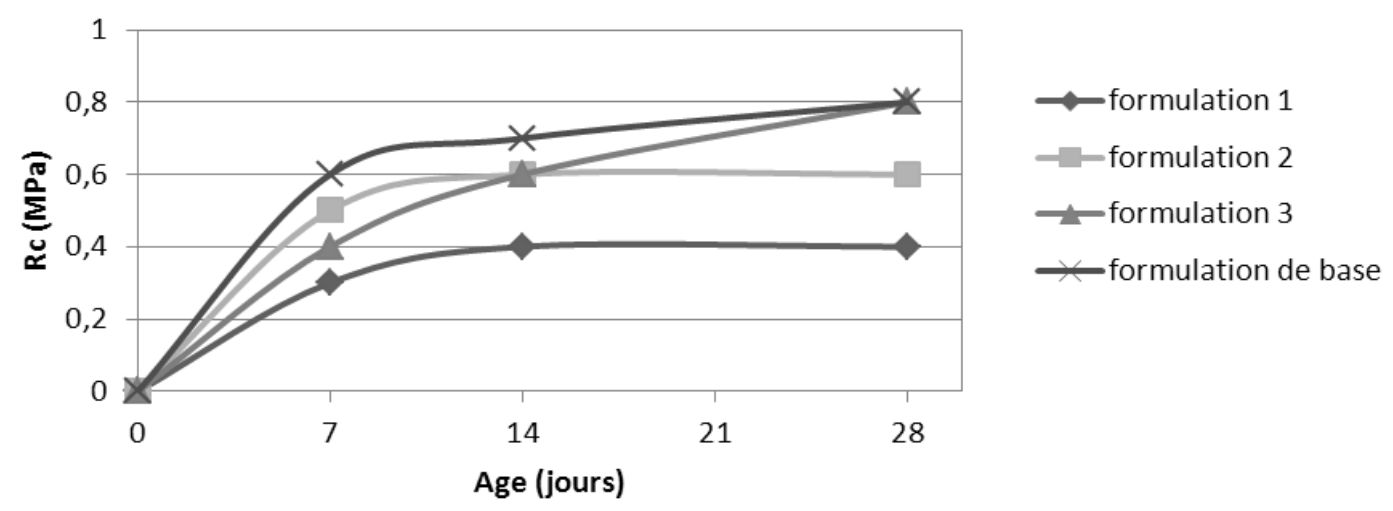

Figure 3. Evolution des résistances à la compression des formulations proposées.

\section{Conclusions}

Une caractérisation géotechnique de base a été effectuée sur des sédiments de Concarneau pour une valorisation en tant que matériau de remplissage. Ces sédiments sont des limons fins très plastiques et organiques à teneur en eau élevée (>200\%). Une valorisation en tant que matériau impose une déshydratation et la présence de matières organiques, l'utilisation de liants hydrauliques et additifs. Le choix des liants s'est porté sur un liant pouzzolanique, de la chaux et du ciment faiblement dosé comparativement à la formulation de base du CCTP. Des formulations ont été proposées et testées sur des éprouvettes réalisées en laboratoire. L'indicateur mécanique retenu $R c_{28}$, résistance à la compression simple à 28 jours, a montré que l'une des formulations, référencée 3, fournissait une valeur de résistance de $0.8 \mathrm{MPa}$, voisine de celle de la formulation du CCTP, respectivement avec un taux de ciment de $3 \%$ et $20 \%$. On observe que les formulations 2, 3 et celle de base présentent des valeurs de $R c$ supérieures à $0.5 \mathrm{MPa}$, suffisamment élevées pour un remplissage dans une zone de stockage.

Par ailleurs les formulations 2 et 3 n'utilisent que 12\% de liants ajoutés par rapport aux $20 \%$ de ciment de la formulation du CCTP pour atteindre des résistances comprises entre 0.6 et $0.8 \mathrm{MPa}$. On remarque aussi l'absence de palier pour les formulations 3 et de base qui contiennent les taux les plus élevés en ciment.

Des essais complémentaires sont nécessaires pour confirmer les tendances observées et optimiser le rapport ciment/liant pouzzolanique. Des essais à long terme seraient utiles pour suivre l'effet de ces liants pouzzolaniques. 
La connaissance de la Mer :

un vecteur du développement durable en Méditerranée

\section{Références bibliographiques}

BEHMANESH J. (2008). Etude de la durabilité d'un sédiment, traité au ciment et additifs. Ph.D Thesis, Université de Caen, 215 p.

BOUTOUIL M. (1998). Traitement des vases de dragage par solidification/ stabilisation à base de ciments et additifs. Ph.D Thesis, Université du Havre, 278 p.

COLIN D. (2003). Valorisation de sédiments fins de dragage en technique routière. Ph.D Thesis, Université de Caen, 180 p.

FOUCHER J.M. (2003). Etude de valorisation des sédiments de dragage du chenal de la Seine en technique routière. Mémoire de DESS Génie littoral, portuaire et des estuaires, Université de Caen, 89 p.

MIRAOUI M. (2010). Mise en ouvre d'une démarche de prétraitement et de traitement des sédiments de dragage en vue d'une valorisation dans le génie civil. Ph.D Thesis, Université des sciences et technologies de Lille 1.

OBANA M., LEVACHER D., DHERVILLY P. (sous presse). Durability properties of marine sediment stabilized by pozzolan and alkali activated binders. European Journal of Environmental and Civil Engineering.

REY F. (1999). Etude physico - mécanique et environnementale du composite vasechaux- additifs. Ph.D Thesis, Université de Caen, 272 p.

SILITONGA E., LEVACHER D., MEZAZIGH S. (2009). Effects of the use of fly ash as a binder on the mechanical behaviour of treated dredged sediments. Environmental Technology, Volume 30, Issue 8, Ed. Thomas Telford, pp 799-807.

SILITONGA E., LEVACHER D., MEZAZIGH S. (2010). Utilization of fly ash for stabilization of marine dredged sediments. European Journal of Environmental and Civil Engineering, volume 14, n²/2010, pp 253-265. doi:10.3166/ejece.14.253-265

SILITONGA E., SHRIVASTAVA A., LEVACHER D. (2008). Influence of fly ash addition on the mechanical properties of treated dredged material. $1^{\text {st }}$ International Symposium on Sediment Management, I2SM, Lille, July 9-11th, pp 243-252. 